\title{
Original
}

\section{Cortical Bone Response Towards Porous Composites of PLGA and Apatite Prepared from Calcium Complexes}

Tohru Hayakawa ${ }^{1)}$, Chihiro Mochizuki ${ }^{2)}$, Hiroki Hara ${ }^{2)}$, Tsuyoshi Amemiya ${ }^{3)}$, Satoshi Hirayama ${ }^{4}$, Fei Yang', Hong Shen', Shenguo Wang', Yoshiki Hamada ${ }^{3)}$ and Mitsunobu Sato' ${ }^{2)}$

\author{
${ }^{1)}$ Department of Dental Engineering, Tsurumi University School of Dental Medicine, Yokohama, Japan \\ ${ }^{2}$ Coordination Engineering Laboratory, Faculty of Engineering, Kogakuin University, Tokyo, Japan \\ ${ }^{3)}$ Department of Oral and Maxillofacial Surgery, Tsurumi University School of Dental Medicine, Yokohama, Japan. \\ 4) Department of Operative Dentistry, Nihon University School of Dentistry at Matsudo, Chiba, Japan \\ ${ }^{5}$ PPCL, Institute of Chemistry, Chinese Academy of Sciences, Beijing 100190, China \\ (Accepted for publication, June 20, 2012)
}

\begin{abstract}
Apatites were prepared by varying the ligands of calcium complexes. Two lower crystallinity apatites were prepared from calcium-iminodiacetic acid (Ca-IDA) or calcium- aspartic acid (Ca-Asp) complexes and one higher crystallinity apatite from Ca-IDA complex. Cortical bone responses towards their PLGA composites including different apatites, namely, PLGA/IDA-HA(lower), PLGA/IDA-HA(higher) and PLGA/Asp-HA(lower), were evaluated after implantation into the cortical bone of rabbit tibiae.

Micro-CT observation showed no distinct differences among the three PLGA/apatite composites after 12 weeks of implantation. The original drill holes were partly closed and formation of primary woven bone was observed. Residue of decomposed PLGA was still observed inside the bone marrow.

The histological appearances of porous PLGA/apatite composite scaffolds after 12 weeks of implantation showed similar overall cortical bone response to different PLGA/apatite composites. The original drill hole was not completely filled with new bone. For PLGA/IDA-HA(higher) composite, the original bone defect was partly filled with new bone. Residue of degraded PLGA and remaining apatite was recognized. Both PLGA/ IDA-HA(lower) and PLGA/Asp-HA(lower) showed residue of degraded PLGA inside the bone marrow. Remnant lower-crystallinity apatite was not clearly recognized.

In conclusion, apatites with different crystallinity were prepared through Ca-IDA and Ca-Asp complexes. The bone responses towards PLGA/apatite composites with different crystallinity were similar, but the degradation behavior of PLGA/apatite composite was different. It is suggested that the degradation of PLGA in PLGA/ apatite composite influences new bone formation in addition to the release and dissolution of calcium from apatite.
\end{abstract}

Keywords: Apatite, PLGA,Porous composite scaffold, Crystallinity, Cortical bone response

\section{Introduction}

Composite materials made from calcium phosphate compounds such as hydroxyapatite and tricalcium phosphate (TCP) and biodegradable polymers such as poly(lactic) acid, poly(glycolic) acid, and poly(lactide-co-glycolide) (PLGA) have been proposed to overcome the shortcomings in the mechanical properties of calcium phosphate such as brittleness and insufficient strength. These composite materials have been used as bone substitutes, and numerous studies reported better biocompatibility and enhancement of osteoconductivity ${ }^{1-7)}$.

Correspondence to: Dr. Tohru Hayakawa, Department of Dental Engineering, Tsurumi University School of Dental Medicine, 2-1-3, Tsurumi, Tsurumi-ku, Yokohama, 230-8501, Japan TEL: +814558083654, FAX: +81-455739599,E-mail: hayakawa-t@tsurumiu.ac.jp
Few studies have reported the relationship between the crystallinity of apatite and bone formation response in vivo. In our previous work, porous composites of PLGA and apatite with different crystallinities were prepared and evaluated for in vivo and in vitro biocompatibility ${ }^{8-10)}$. Two types of apatite, a low crystallinity calcium-deficient hydroxyapatite (CDHA) and a higher crystallinity apatite mixture of carbonate hydroxyapatite and CDHA, were prepared from calcium-ethylenendiamine tetraacetic acid (Ca-EDTA) complex. PLGA/apatite porous composite with a lower crystallinity showed faster degradation in phosphate buffered saline solution and more rapid and greater amount of calcium phosphate precipitation in simulated body fluid. Moreover, porous PLGA/apatite composite with lower crystallinity produced a significantly greater amount of new bone after 


\begin{tabular}{|c|c|c|}
\hline ligand & Structural formulae & $\begin{array}{c}\text { Stability constant } \\
\text { of Ca-complex }\end{array}$ \\
\hline IDA & $\mathrm{HN}<\begin{array}{l}\mathrm{CH}_{2} \mathrm{COOH} \\
\mathrm{CH}, \mathrm{COOH}\end{array}$ & 2.59 \\
\hline Asp & $\begin{array}{c}\mathrm{COOH} \\
\mathrm{H}_{2} \mathrm{~N}-\stackrel{\mathrm{CH}}{!} \\
\text { CH} \\
\text { । } \\
\mathrm{COOH}\end{array}$ & 1.67 \\
\hline EDTA & $-\mathrm{CH}_{2} \mathrm{CH}_{2}-\mathrm{N}$ & 10.61 \\
\hline
\end{tabular}

Figure1. Structural formulae of IDA, Asp and EDTA and the stability constant of each calcium complex

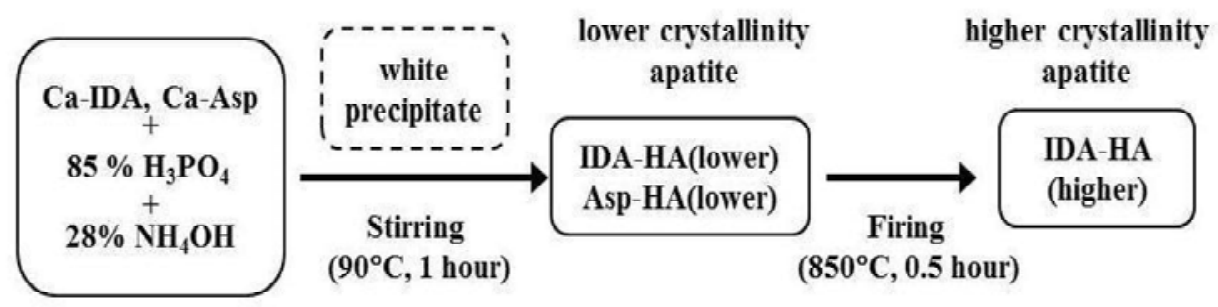

Figure 2. Schematic drawing of apatite preparation

implantation into cortical bone defects of rabbits ${ }^{8-10)}$.

The advantages of apatite synthesis through Ca-EDTA complex are the homogeneous reaction mixture, easy control of $\mathrm{Ca} / \mathrm{P}$ ratio and easy control of apatite crystallinity by regulating the reaction conditions. However, the apatite with lower crystallinity originating from Ca-EDTA complex was calcium-deficient. Also, a large amount of $\mathrm{H}_{2} \mathrm{O}_{2}$ was needed for the decomposition of CaEDTA complex and the yield for CDHA was very low, approximately $32 \%$. It is presumed that stability constant of the calcium complex affects the decomposition of complex and the yield of apatite. Easier decomposition of calcium complex with a lower stability constant is expected to produce apatite which is not calcium deficient.

Recently, Mochizuki et al. reported that lower-crystallinity apatite could be prepared by varying the ligands of calcium complexes ${ }^{11}$. Calcium complexes of iminodiacetic acid (IDA) or aspartic acid (Asp), the stability constants of which are smaller than that of EDTA, were employed. In the present study, two lower crystallinity apatites were prepared from Ca-IDA or Ca-Asp complexes and one higher crystallinity apatite was prepared from Ca-IDA complex. In vivo bone responses towards their PLGA composites including different apatites were evaluated after implantation into the cortical bone of rabbit tibiae.

\section{Materials and Methods}

\section{Apatite preparation}

Apatites were prepared via the Ca-IDA or Ca-Asp complexes. The structural formulations of IDA and Asp are shown in Fig.1 along with EDTA. The stability constants of their Ca complex are also listed. The synthetic scheme for apatites is shown in Fig.2. Three kinds of apatite, IDA-HA(lower), IDA-HA(higher) and Asp$\mathrm{HA}\left(\right.$ lower) were prepared according to the previous report ${ }^{11)}$.

\section{IDA-HA(lower): lower crystallinity apatite from Ca complexes of IDA}

A mixed solution of Ca-IDA complex and $\mathrm{H}_{3} \mathrm{PO}_{4}$ in water was heated to $90^{\circ} \mathrm{C}$ with stirring. After addition of $\mathrm{NH}_{4} \mathrm{OH}$ to the hot solution, white powders immediately precipitated. The solution was stirred at $90^{\circ} \mathrm{C}$ for $1 \mathrm{~h}$, and IDA-HA(lower) powder was isolated after the cooling.

\section{IDA-HA(higher):higher crystallinity apatite from calcium complexes of IDA}

IDA-HA(lower) powder was heated at $850^{\circ} \mathrm{C}$ for 0.5 hours, and then IDA-HA(higher ) power was obtained.

Asp-HA(lower): lower crystallinity apatite from calcium complexes of Asp 


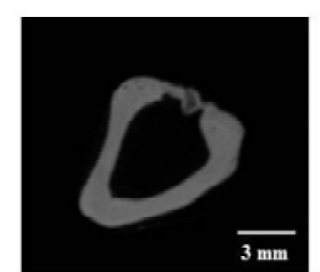

PLGA/IDA-HA(higher)

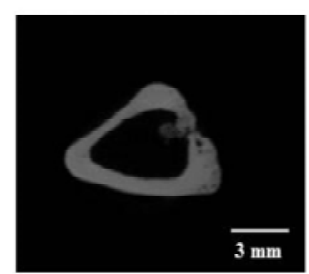

PLGA/IDA-HA(lower)

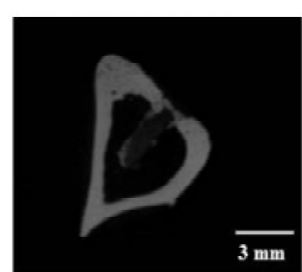

PLGA/Asp-HA(lower)

Figure 3. Micro-CT views of rabbit tibiae after 12 weeks of implantation of porous PLGA/ apatite composite.

L-Asp, $\mathrm{Ca}(\mathrm{OH})_{2}$ and $\mathrm{H}_{2} \mathrm{O}$ were mixed, and then $\mathrm{H}_{3} \mathrm{PO}_{4}$ was added. The obtained $\mathrm{Ca}-\mathrm{Asp}-\mathrm{PO}_{4}$ solution was heated to $90^{\circ} \mathrm{C}$ with stirring. After addition of $\mathrm{NH}_{4} \mathrm{OH}$ to the solution, white powders were immediately precipitated, and then the solution was stirred for $1 \mathrm{~h}$ at $90^{\circ} \mathrm{C}$. Asp-HA(lower) powder was isolated from the reacted solution.

\section{PLGA and porous PLGA/apatite composite preparation}

PLGA (70/30: $\left.\mathrm{Mw}=11.5 \times 10^{4}\right)$ was prepared via ring-opening co-polymerization of L-lactide (PURAC, the Netherlands) and glycolide (PURAC, the Netherlands) at a molar ratio of 70:3012).

Three kinds of PLGA porous composites (50-/50 mass\%) including different apatites, PLGA/IDA-HA(lower), PLGA/IDAHA(higher) and PLGA/Asp-HA(lower), were fabricated by the solution casting/particle leaching method as described elsewhere ${ }^{8,13)}$. Porous PLGA composites were kept in a dry vacuum until use.

\section{Implantation procedure}

The animal study was conducted in accordance with the animal experimental ethical guidelines of Tsurumi University School of Dental Medicine (No.091). Six 3-month-old female Japanese White Rabbits weighing about 3.5-4kg were used. Porous PLGA/ IDA-HA(higher), PLGA/IDA-HA(lower) and PLGA/AspHA(lower) composites were inserted into the cortical bone of rabbits according to a previously reported technique ${ }^{9,10)}$. Each animal received two types of porous PLGA composites. Namely, two rabbits received PLGA/IDA-HA(higher) and PLGA/IDAHA(lower) composite in each tibia, respectively. Another two rabbits received PLGA/IDA-HA(lower) and PLGA/Asp$\mathrm{HA}$ (lower) composite, and the remaining two rabbits received PLGA/Asp-HA(lower) and PLGA/IDA-HA(higher) in each tibia, respectively. Thus, the number of implants of each material, PLGA/ IDA-HA(higher), PLGA/IDA-HA(lower) and PLGA/AspHA(lower) composite, was four. Before surgery, all PLGA/apatite composites were sterilized with ethylene-oxide gas.

Surgery was performed under general inhalation anesthesia with a $4 \%$ isoflurane and oxygen mixture, which was reduced to $2 \%$ isoflurane during surgical manipulation. Local anesthesia was administered by xylocain injection. To reduce the perioperative infection risk, a prophylactic antibiotic, Shiomalin ${ }^{\circledR}$ (equivalent to Latamoxef Sodium, Shionogi \& Co., Ltd, Japan), was administered postoperatively by subcutaneous injection.

Porous PLGA/apatite composites ( $4.5 \mathrm{~mm} \times 3.5 \mathrm{~mm} \times 2 \mathrm{~mm}$ ) were inserted into the left and right tibial diaphyses of the rabbits. For insertion of the porous material, each animal was immobilized on its back. The hind legs of the rabbits were shaved, washed, and disinfected with iodine tincture. A longitudinal incision was made along the medial surface of the left and right tibiae, and the bone was exposed by blunt dissection. Cortical bone defects measuring $2 \times 4 \mathrm{~mm}$ were created through the medial cortex and the medulla. These defects were prepared with a very gentle surgical technique using a low rotational drilling speed $(500 \mathrm{rpm})$ and continuous internal cooling. After press-fit insertion, the soft tissues were closed in separate layers using restorable Vicryl 3-0 sutures.

Postoperatively, the animals were placed in a standard cage. They were fed with water and rabbit diet ad libitum and were allowed to move unrestricted at all times. The rabbits were sacrificed by peritoneal injection of an overdose of thiamyral sodium (Isozol ${ }^{\circledR}$ ) after 12 weeks of implantation.

\section{Micro CT and histological observation}

Each PLGA/apatite composite and surrounding bone was excised immediately after sacrifice. Excess tissue of the excised specimen was then removed and fixed in $10 \%$ buffered formalin solution. Afterwards, tissue blocks containing PLGA/apatite composites were dehydrated through a graded series of ethanol and embedded in methylmethacrylate. After polymerization at $37^{\circ} \mathrm{C}$, the embedded samples were obtained.

Before polymerization, the healing condition of the defects in the tibia was observed using high-resolution microfocus X-ray computed tomography (micro-CT, Latheta, LCT-100, HitachiAloka Medical, Ltd, Tokyo, Japan) with a voltage of $50 \mathrm{kV}$, a tube current of $1 \mathrm{~mA}$, and a slice thickness of $0.1 \mathrm{~mm}$. For micro CT observation, three dehydrated specimens for each material were randomly selected and observed.

After micro CT observation, non-decalcified thin sections were prepared using a cutting-grinding technique (EXAKT-Cutting Grinding System, BS-300CP band system \& 400 CS microgrinding system, EXAKT) ${ }^{14)}$. Sections with a final thickness of 

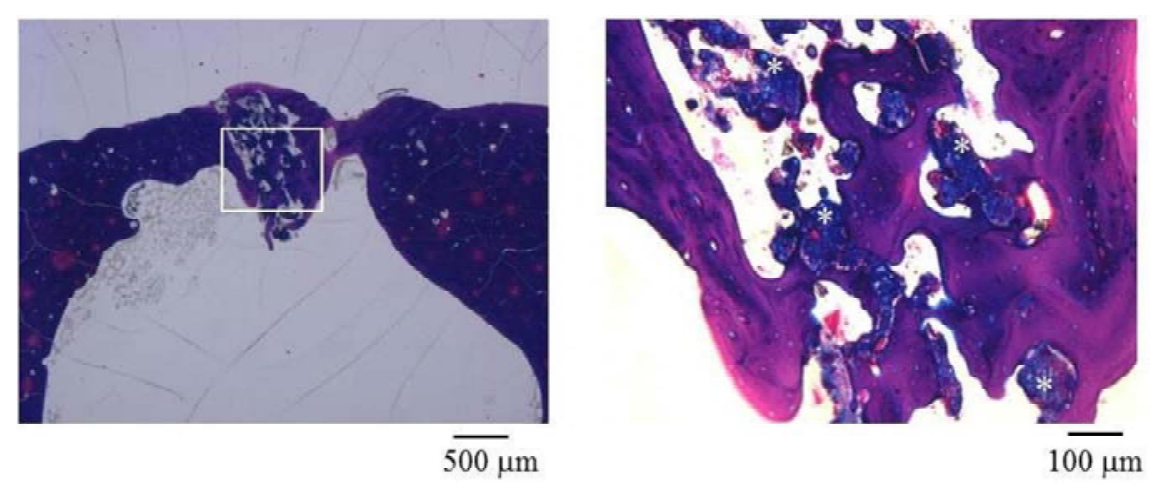

Figure 4. The histological appearance after 12 weeks of implantation of porous PLGA/ IDA-HA(higher) composite *: remnant apatite
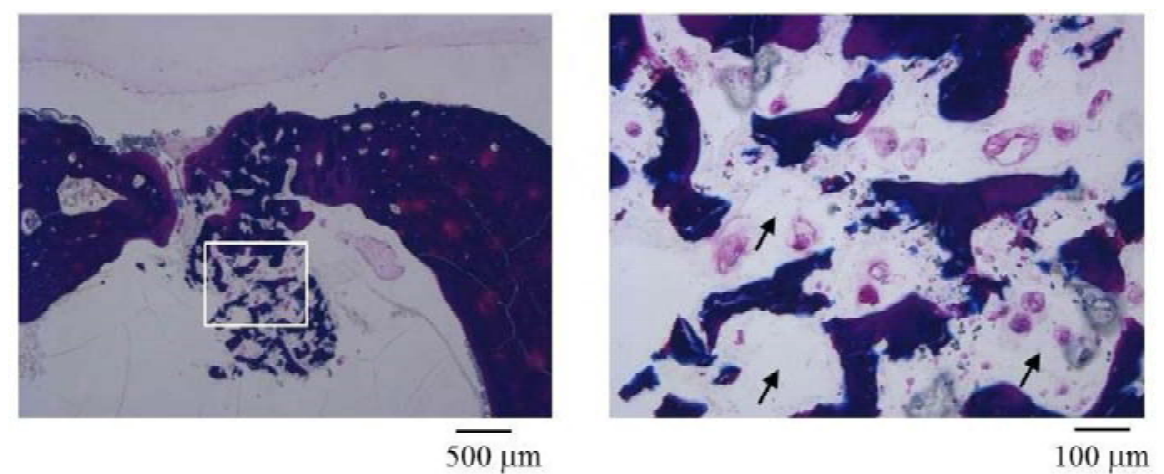

Figure 5. The histological appearance after 12 weeks of implantation of porous PLGA/ IDA-HA(lower) composite. Arrow: apatite has dissolved
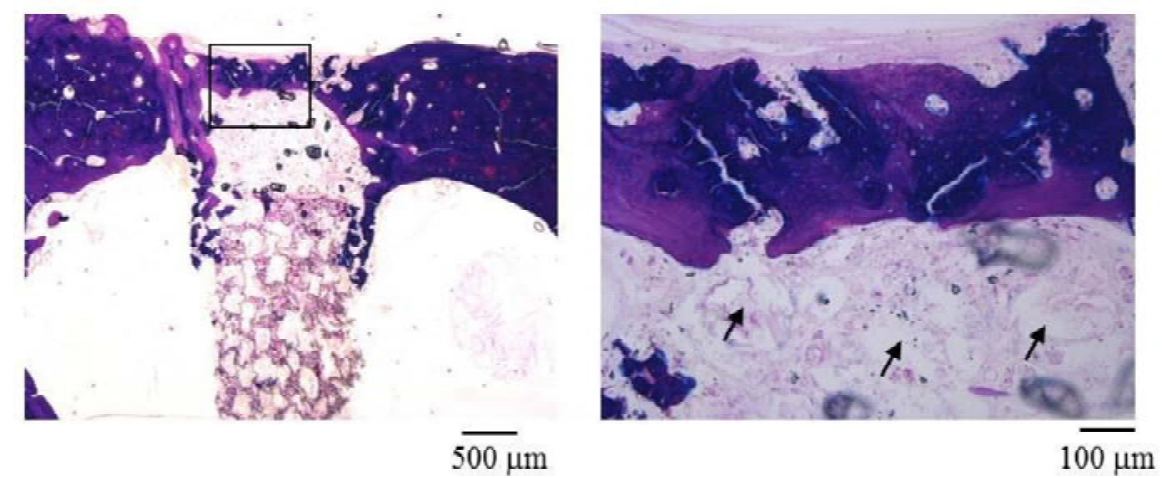

Figure 6.The histological appearance after 12 weeks of implantation of porous PLGA/ Asp-HA(lower) composite Arrow: apatite has dissolved

approximately $50 \mu \mathrm{m}$ were made in a direction parallel to the long axis of the implanted composites and were stained with methylene blue and basic fuchsin. The bone response towards the composite materials was evaluated using a light microscope (BX51, Olympus Corp., magnification x 100).

\section{Results}

During the test period, the experimental animals remained in good health. At sacrifice, no clinical signs of inflammation or adverse tissue reactions were seen.
Figure 3 shows the micro-CT appearance of the rabbit tibiae after 12 weeks' implantation of porous PLGA/HA composite scaffold implantation. No distinct differences were observed among the three PLGA/apatite composites. The original drill holes were partly closed and formation of primary woven bone was observed. Residue of decomposed PLGA was still observed inside the bone marrow.

The histological appearances of porous PLGA/apatite composite scaffolds after 12 weeks of implantation are shown in Figures 4-6. No inflammatory reactions were seen in any samples, 
Tohru Hayakawa et al.: Bone Response Towards Porous Composite of PLGA and Apatite

and no clear appearances of macrophages or foreign-body giant cells were recognized in any specimen. The overall cortical bone responses to the different PLGA/apatite composites were similar and their histological appearance corresponded with the results of the micro-CT observation.

For PLGA/IDA-HA(higher) composite, the original bone defect was partly filled with new bone, and residue of degraded PLGA was recognized inside the bone marrow (Fig. 4a). Higher magnification clearly identified the remnant higher-crystallinity apatite (asterisk) with along with the new bone (Fig. 4b).

The original drill holes were also not completely filled with new bone for both PLGA/IDA-HA(lower) and PLGA/AspHA(lower) (Figs. 5\&6). Residue of degraded PLGA was clearly observed inside the bone marrow and the part where apatite had disappeared was clearly identified (arrow head). Especially, PLGA/ Asp-(lower) showed the original PLGA composite shape (Fig. 6). Remaining lower-crystallinity apatite with was not clearly recognized for either PLGA/apatite composite, even under higher magnification.

\section{Discussion}

In the present study, we evaluated the cortical bone response towards PLGA/apatite composite scaffolds with different levels of crystallinity.

The apatite employed in the present study was prepared from Ca complex with IDA or Asp. Previous studies reported apatite synthesis from Ca-EDTA complex. Ca-IDA and Ca-Asp complex have smaller stability constants than Ca-EDTA complex, as shown in Fig.1. Ca-IDA and Ca-Asp complexes could provide higher yields of apatite than Ca-EDTA complex and could produce apatite which was not calcium-deficient.

There are few reports about the influence of apatite crystallinity on bone response. Regarding apatite-coated titanium implants, it was reported that an apatite-coated titanium implant with $98 \%$ crystallinity exhibited inferior bone bioactivity to a $55 \%$ crystalline apatite coating ${ }^{15)}$. It is speculated that the release and dissolution of calcium from apatite stimulate osteoblast-like cells and enhance bone formation. An alternative speculation is that the release and dissolution of calcium of apatite will produce a supersaturated condition and provide calcium deposition ${ }^{16,17)}$. In contrast, Pathi et al. prepared apatite nanoparticles with a narrow size distribution and varying crystallinity and suggested that low-crystallinity apatite nanoparticles promote greater adsorption of adhesive serum proteins and enhance breast tumor adhesion while more crystalline apatite nanoparticles stimulate enhanced expression of the osteolytic factor interleukin- $8^{18)}$.

Our previous studies ${ }^{9,10)}$ demonstrated that the level of apatite crystallinity influences bone response, and the PLGA/apatite composite with a lower level of apatite crystallinity produced a significantly greater amount of new bone. Cortical bone defects of rabbit tibia were almost completely filled with new bone. However, the present study showed no distinct differences of bone formation between porous composites of PLGA and apatites with different levels of crystallinity.

The degradation behaviors of PLGA/apatite composites were different between lower- and higher-crystallinity apatite. Remnant apatite was clearly recognized for PLGA/IDA-HA(higher) composite, while apatite was dissolved for PLGA/IDA-HA(lower) and PLGA/Asp-HA(lower) composites. Lower-crystallinity of apatite is easier to dissolve in vivo. It is presumed that the dissolution of apatite is faster than the degradation of PLGA for PLGA/IDA-HA(lower) and PLGA/Asp-HA(lower) composites. Although the detailed mechanism of bone formation is not still clear, it is possible that the faster dissolution of apatite with lower crystallinity caused the insufficient bone formation of PLGA/ apatite composite.

The balance between the release and dissolution rate of calcium and bone formation rate is the key factor for the bone healing process. The dissolution of calcium is controlled by the crystallinity of apatite, as mentioned above. Moreover, the release of calcium from apatite is influenced by the degradation of PLGA. Remnant PLGA was clearly identified and PLGA had not completely decomposed after 12 weeks of implantation. These results differed from our previous experiments ${ }^{9,10)}$. In the present study, remnant PLGA might have inhibite new bone formation. The relationship between the degradation behavior of PLGA and bone formation will need to be further investigated in detail.

In the present study, two lower crystallinity apatites were prepared using different ligands, but no clear differences in bone response were observed. The content of apatite in a PLGA/apatite composite will also influence the bone formation. More content of lower crystallinity apatite or less content of higher crystallinity apatite is expected to promote the bone healing process and enhance the new bone formation.

In conclusion, apatites with different crystallinity were prepared through Ca-IDA and Ca-Asp complexes. The bone responses towards PLGA/apatite composites with different crystallinity were similar, but the degradation behaviors of PLGA/apatite composites were different. It is suggested that the degradation of PLGA in PLGA/apatite composite influences new bone formation.

\section{Acknowledgements}

A part of this study was supported by Grants-in-Aid for Scientific Research (B) (23390453) from the Japan Society for the Promotion of Science.

\section{References}

1. Wei G and Ma PX. Structure and properties of nanohydroxyapatite/polymer composite scaffolds for bone tissue engineering. Biomaterials 25: 4749-4757, 2004

2. Jayasuriya AC, Assad M, Ebraheim NA and Jayatissa AH. 
Dissolution behavior of biomimetic minerals on 3D PLGA scaffold. Surf Coating Tech 200: 6336-6339, 2006

3. Kim SS, Sun Park M, Jeon O, Cha Yong C and Kim BS. Accelerated bonelike apatite growth on porous polymer/ ceramic composite scaffolds in vitro. Tissue Engin 12: 29973006, 2006

4. Leong NL, Jiang J and Lu HH. Polymer-ceramic composite scaffold induces osteogenic differentiation of human mesenchymal stem cells. Conf Proc IEEE Eng Med Biol Soc 1: 2651-2654, 2006.

5. Simpson RL, Wiria FE, Amis AA, Chua CK, Leong KF, Hansen UN, Chandrasekaran M and Lee MW. Development of a 95/5 poly(L-lactide-co-glycolide)/ hydroxylapatite and b-tricalcium phosphate scaffold as bone replacement material via selective laser sintering. J Biomed Mater Res. Part B Appl Biomater 84: 17-25, 2008

6. Zheng L, Yang F, Shen H, Hu X, Mochizuki C, Sato M, Wang S and Zhang Y. The effect of composition of calcium phosphate composite scaffolds on the formation of tooth tissue from human dental pulp stem cells. Biomaterials 32 : 7053-7059, 2011.

7. Daculsi G, Goyenvalle E, Cognet R, Aguado E and Suokas EO. Osteoconductive properties of poly(96L/4D-lactide)/ beta-tricalcium phosphate in long term animal model. Biomaerials 32: 3166-3177, 2011.

8. Mochizuki C, Sasaki Y, Hara H, Sato M, Hayakawa T, Yang F, Hu X, Shen H and Wang S. Crystallinity control of apatite through Ca-EDTA complexes and porous composites with PLGA. J Biomed Mater Res Part B 90B: 290-301, 2009.

9. Hayakawa T, Mochizuki C, Hara H, Fukushima T, Sato M, Yang F, Shen $\mathrm{H}$ and Wang S. Influence of apatite crystallinity in porous PLGA/apatite composite scaffold on cortical bone response. J Hard Tissue Biology 18: 7-12, 2009.

10. Hayakawa T, Mochizuki C, Hara H, Sato M, Yang F, Shen H and Wang S. In vivo evaluation of composites of PLGA and apatite with two different levels of crystallinity. J Mater Sci: Mater Med 21: 251-258, 2010.

11. Mochizuki C, Hayakawa T, Wang SG and Sato M. In vitro examination of apatite/PLGA composite including low crystallinity apatites prepared from Ca complex of IDA and Asp. $18^{\text {th }}$ International SPACC Symposium.

12. Cai Q, Bei JZ, Luo AQ and Wang SG. Biodegradation behavior of poly(lactide-co-glycolide) induced by microorganisms. Polym Degrad Stabil 71: 243-251, 2001

13. Shi GX, Cai Q, Wang CY, Lu N, Wang SG and Bei JZ. Fabrication and biocompatibility of cell scaffolds of poly(Llactic acid) and poly(L-lactic-co-glycolic acid). Polym Adv Technol 13: 227-232, 2002.

14. Donath K and Breuner G. A method for the study of undecalcified bones and teeth with attached soft tissues. The Sage-Schliff (sawing and grinding) technique, J. Oral Pathol 11: 318-326, 1982.

15. Xue W, Liu X, Zheng X and Ding C. Effect of hydroxyapatite coating crystallinity on dissolution and osseointegration in vivo. J Biomed Mater Res 74A: 553-561, 2005

16. Masuda T, Yliheikkilä PK, Felton DA and Cooper LF. Generalizations regarding the process and phenomenon of osseointegration. Part I. In vivo studies. Int J Oral Maxillofac Implants 13: 17-29, 1998.

17. Cooper LF, Masuda T, Yliheikkilä PK and Felton DA. Generalizations regarding the process and phenomenon of osseointegration. Part II. In vitro studies. Int J Oral Maxillofac Implants 13: 163-174, 1998.

18. Pathi SP, Lin DD, Dorvee JR, Estroff LA and Fischbach C. Hydroxyapatite nanoparticle-containing scaffolds for the study of breast cancer bone metastasis. Biomaterials. 32; 5112-5122: 2011 
